\title{
UBIAD1 expression is associated with cardiac hypertrophy in spontaneously hypertensive rats
}

\author{
BINGJU YAN $^{1}$ and JUN WANG ${ }^{2}$ \\ Departments of ${ }^{1}$ Cardiology and ${ }^{2}$ General Surgery, First Hospital of Jinzhou Medical University, \\ Jinzhou, Liaoning 121000, P.R. China
}

Received December 18, 2017; Accepted October 5, 2018

DOI: $10.3892 / \mathrm{mmr} .2018 .9693$

\begin{abstract}
The present study investigated the potential role of UbiA prenyltransferase domain-containing 1 (UBIAD1) in the pathogenesis of hypertensive cardiac hypertrophy. Spontaneously hypertensive rats (SHRs) and Wistar-Kyoto (WKY) rats at 8, 16 and 28 weeks of age were used. Blood pressure was measured using a non-invasive tail cut-off system. Cardiac functional index was assessed by arterial catheterization. Myocardial structure and cell apoptosis were evaluated by hematoxylin and eosin staining, and terminal deoxynucleotidyl-transferase-mediated dUTP nick end labeling assays, respectively. Myocardial expression of UBIAD1, coenzyme Q10 (CoQ10), endothelial nitric oxide synthase (eNOS) and atrial natriuretic peptide were evaluated by immunohistochemistry, western blotting and reverse transcription-quantitative polymerase chain reaction. Circulating and myocardial expression of nitric oxide (NO) were measured using the Griess method. SHRs exhibited increased blood pressure and cardiomyocyte apoptosis, as well as cardiac hypertrophy, compared with age-matched WKY rats. Myocardial expression of UBIAD1 was significantly decreased in SHRs in an age-dependent manner. Similarly, myocardial CoQ10 and eNOS expression were significantly reduced in SHR compared to age-matched WKY rats, and these expression levels additionally decreased further with aging. Serum and myocardial NO expression was additionally decreased in SHRs. Decreased UBIAD1 expression in SHR hearts was associated with decreased levels of CoQ10, eNOS and NO. Given the well-established role of UBIAD1 in the regulation of NO signaling, reduced expression of UBIAD1 in SHR hearts potentially contributed to the pathogenesis of hypertensive cardiac hypertrophy. Therefore, UBIAD1 may
\end{abstract}

Correspondence to: Dr Jun Wang, Department of General Surgery, First Hospital of Jinzhou Medical University, 2 Renmin Street, Jinzhou, Liaoning 121000, P.R. China

E-mail: wangjunybj@163.com

Key words: UbiA prenyltransferase domain-containing protein 1, spontaneously hypertensive rats, cardiac hypertrophy, coenzyme Q10, endothelial nitric oxide represent a potential therapeutic target for clinical treatment of hypertensive cardiac hypertrophy.

\section{Introduction}

UbiA prenyltransferase domain-containing protein 1 (UBIAD1), additionally referred to as transitional epithelial response gene, was first identified as a tumor suppressor in bladder cancer (1-3). The UBIAD1 gene is located on chromosome 1p36 and encodes a protein of 338 amino acids, harboring the UbiA isopentyl transferase domain (1). Previous studies demonstrated that UBIAD1 exhibits specific subcellular localization, and is expressed in the mitochondria (4), Golgi (5) and endoplasmic reticulum (6). UBIAD1 is conserved across different species, including zebrafish and humans (7). UBIAD1 converts menadione to MK-4, a principal form of vitamin $\mathrm{K}$ in humans (6). In addition, UBIAD1 catalyzes the non-mitochondrial coenzyme Q10 (CoQ10) in zebrafish (5), which serves an important role in producing endothelial nitric oxide (eNOS) and nitric oxide (NO) (8). Therefore, UBIAD1 has critical functions in maintaining cellular homeostasis.

UBIAD1 has been demonstrated to be involved in a variety of human diseases. For instance, naturally occurring mutations in the UBIAD1 gene have been causally linked to Schneider lens corneal dystrophy, a genetic autosomal dominant disease that is caused by abnormal cholesterol and phospholipid metabolism (2). Furthermore, UBIAD1 functions as a modifier of serine/threonine-protein kinase PINK1, mitochondrial, and this mutation is associated with Parkinson's disease (9). UBIAD1 may also act as a tumor suppressor through negatively regulating the Ras-mitogen activated protein kinase (MAPK) signal transduction pathway $(1,10)$. At present, a limited number of studies have elucidated the role of UBIAD1 in cardiovascular disease. In zebrafish, there is specific evidence that UBIAD1 is cardioprotective against oxidative stress by mediating CoQ10 synthesis (5), and that a UBIAD1 mutant causes cardiac edema (7). Whether UBIAD1 is involved in cardiac pathophysiology in mammals is not yet known.

Cardiac hypertrophy is a principal factor leading to cardiac muscle disorders. The exact mechanisms of cardiac hypertrophy remain unclear; however, multifactorial mechanisms, including genetics and environmental cues, likely serve a role in pathogenesis. Multiple lines of evidence have suggested that NO is involved in the pathophysiology of many diseases, 
including cardiac hypertrophy (11). For instance, increased levels of NO have been reported to reduce cardiomyocyte hypertrophy progression (12). Similarly, overexpression of eNOS was demonstrated to inhibit cardiac hypertrophy in mice (13); however, suppression of eNOS activity promotes cardiac hypertrophy, as evidenced by eNOS deficient mice (14). In addition, decreased levels of eNOS are associated with angiotensin II receptor knockout-induced cardiac hypertrophy (15). Collectively, these findings suggest the important roles of eNOS-NO signaling in the pathogenesis and progression of cardiac hypertrophy.

As mentioned above, UBIAD1 has been associated with a number of human diseases. However, the role of UBIAD1 in cardiac hypertrophy has not yet been investigated. Given that UBIAD1 is an important regulator of eNOS-NO signaling and CoQ10 synthesis, which significantly contribute to the pathogenesis of cardiovascular diseases (16), it was hypothesized that UBIAD1 functions in the development of cardiac hypertrophy. In the present study, the expression of UBIAD1 and its downstream signaling molecules was measured in the hearts of spontaneously hypertensive rats (SHRs) and age-matched control Wistar-Kyoto (WKY) rats using various molecular approaches. The findings of the present study offer novel insight into the mechanisms underlying the development of hypertensive cardiac hypertrophy, and provide novel insight for the prevention and treatment of hypertension.

\section{Materials and methods}

Reagents. UBIAD1 antibody (cat. no. sc-377013) and GAPDH antibody (cat. no. sc-32233) were purchased from Santa Cruz Biotechnology, Inc. (Dallas, TX, USA). CoQ10 antibody (cat. no. 17812-1-AP), eNOS antibody (cat. no. 20116-1-AP) were purchased from Wuhan Sanying Biotechnology (Wuhan, China). Goat-antibody rabbit secondary antibodies (cat. no. A0216) and goat-antibody mouse secondary antibodies (cat. no. A0208) were purchased from Beyotime Institute of Biotechnology (Shanghai, China). Terminal deoxynucleotidyl-transferase-mediated dUTP nick end labeling (TUNEL) and DAB reaction kits were purchased from Beyotime Institute of Biotechnology (Shanghai, China), and the polymerase chain reaction (PCR) kits were purchased from Takara Bio, Inc. (Otsu, Japan).

Animals. A total of 48 eight-week male rats (24 SHR and 24 WKY) were obtained from the Beijing Vital River Laboratory Animal Technology Co. Ltd. (Beijing, China). At the start of the study, the rats were randomly divided into groups for three different ages: 8, 16 and 32 weeks (8 rats/group). After the different periods of time, subsequent experiments were performed. Rats were housed in an environment with a temperature of $22-27^{\circ} \mathrm{C}$, humidity $50 \pm 10 \%$ and $12 / 12 \mathrm{~h}$ light/dark cycle. All rats had free access to water and regular chow. All animal protocols complied with and were approved by the Ethics Committee of Jinzhou Medical University.

Blood pressure measurement. Blood pressure was measured using a non-invasive tail cut-off multi-channel blood measurement device (Xinhua Surgical Instrument Co., Ltd., Zibo, China). Blood pressure measurements were repeated three times, and the mean value was taken following the final blood pressure measurement.

Measurement of cardiac function parameters. Animals were anesthetized with an intraperitoneal injection of chloral hydrate $(300 \mathrm{mg} / \mathrm{kg})$, which was prepared in sodium chloride to a final concentration of $15 \%$. Following the induction of anesthesia, rats were placed on a warming pad. Catheters were inserted into the femoral and subclavian arteries and connected to the multi-conductive physiological recorder pressure transducer (ADInstruments Pty Ltd., Sydney, Australia). Following stabilization, the following cardiac functional parameters were measured: Maximal increase rate of left ventricular pressure $(+\mathrm{dp} / \mathrm{dt}$ max), maximal drop rate of left ventricular pressure (-dp/dt max), left ventricular end diastolic pressure (LVEDP), and left ventricular systolic pressure (LVSP). No signs of peritonitis were observed following anesthesia administration.

Determination of cardiac hypertrophic index. Rats were sacrificed following cardiac function measurements, and body weight $(\mathrm{BW})$ and left ventricular weight (LVW) were subsequently measured. The cardiac hypertrophic index was defined as the ratio of LVW to BW, which represented the degree of ventricular hypertrophy.

Hematoxylin and eosin $(H \& E)$ staining. Rat left ventricular myocardium was collected, fixed in $10 \%$ neutral formic acid solution at room temperature for 24-48 h, embedded in wax and cut into $5 \mu \mathrm{m}$ thick sections. H\&E staining was performed according to a widely used standard protocol; $0.8 \%$ hematoxylin staining for $5 \mathrm{~min}$ at room temperature, and $0.35 \%$ eosin staining for $3 \mathrm{~min}$ at room temperature. Cardiac myocardial structures, including myocardial cell morphology and myocardial fiber arrangement, were viewed under an ordinary light microscope (Olympus BX53; Camera system DP73; magnification, x200).

Immunohistochemistry. Cardiac sections were dewaxed using the following procedure: $60^{\circ} \mathrm{C}$ for $2 \mathrm{~h}$, followed by two rounds of xylene for $15 \mathrm{~min}$ each. Subsequently, sections were placed in the gradient alcohol and prepared with dewaxed water as follows: In anhydrous ethanol I and II with for 5 min each, sequentially in 95, 85 and $75 \%$ ethanol with for 2 min each. Sections were subsequently placed in distilled water for $2 \mathrm{~min}$ and in PBS for $5 \mathrm{~min}$. Antigen retrieval was performed by boiling the dewaxed slides in antigen retrieval buffer $(10 \mathrm{mM}$ sodium citrate; PH 6.0) for $10 \mathrm{~min}$, followed by natural cooling and three washes with PBS (5 min/wash). To quench the non-specific background signals, slides were incubated with hydrogen peroxide $\left(\mathrm{H}_{2} \mathrm{O}_{2}\right)$ for $5 \mathrm{~min}$ at room temperature, then washed three times with PBS. The slides were blocked in $1 \%$ bovine serum albumin (cat. no. A600903; Sangon Biotech Co. Ltd., Shanghai, China) at room temperature for $1 \mathrm{~h}$, washed with PBS, and incubated with an anti-UBIAD1 antibody (1:50 dilution) at $4^{\circ} \mathrm{C}$ overnight. Thereafter, the slides were washed with PBS and incubated with horseradish peroxidase-conjugated donkey anti-goat immunoglobulin G antibody (cat. no. A0181; Beyotime Institute of Biotechnology; 1:200) for $30 \mathrm{~min}$ at $37^{\circ} \mathrm{C}$. Staining was visualized with DAB and observed under an ordinary light microscope (Olympus BX53; Camera system: 
DP73; magnification, x200). In total, five randomly selected fields per section were scored and quantified using Image-Pro Plus 5.0 image analysis software (National Institutes of Health, Bethesda, MD, USA).

TUNEL staining. Myocardial cell apoptosis was assessed by TUNEL staining according to the protocol provided by the manufacturer (Beyotime Institute of Biotechnology). The number of apoptotic cardiomyocytes was scored in five randomly selected fields under a microscope (magnification, $\mathrm{x} 400$ ). Apoptotic index was calculated as the number of apoptotic cells/number of cardiomyocytes x100.

Reverse transcription-quantitative PCR (RT-qPCR). Total RNA was extracted from the left ventricles with TRIzol ${ }^{\circledR}$ reagent (Thermo Fisher Scientific, Inc., Waltham, MA, USA) according to the manufacturer's protocol. Purified RNA was dissolved in DEPC water and frozen at $-80^{\circ} \mathrm{C}$ following the determination of RNA concentration. RNA $(1 \mu \mathrm{g})$ was used for the RT reaction in a final volume of $20 \mu \mathrm{l}$ using PrimeScript RT Enzyme Mix (Takara Bio, Inc.) at $37^{\circ} \mathrm{C}$ for $15 \mathrm{~min}$ and $85^{\circ} \mathrm{C}$ for $5 \mathrm{sec}$. All primers were designed based on gene sequences using primer 5.0 software (Primer Premier 5.0; Premier Biosoft International, Palo Alto, CA USA), and the primer sequences used are listed in Table I. qPCR was performed using SYBR Premix Ex Taq (Takara Bio, Inc.) in a final volume of $50 \mu \mathrm{l}$ on an ABI7500 amplifier. The PCR conditions were as follows: $95^{\circ} \mathrm{C}$ for $30 \mathrm{sec}, 40$ cycles of $95^{\circ} \mathrm{C}$ for $5 \mathrm{sec}, 60^{\circ} \mathrm{C}$ for $30 \mathrm{sec}$ and $72^{\circ} \mathrm{C}$ for $30 \mathrm{sec}$, and $4^{\circ} \mathrm{C}$ for $5 \mathrm{~min}$. Relative quantification was performed using the reference gene GAPDH as an internal control. The relative expression was assessed and converted to fold changes using the $2^{-\Delta \Delta \mathrm{Cq}}$ method (17).

Western blotting. Total protein was purified from rat hearts in radioimmunoprecipitation assay lysis buffer (Beyotime Institute of Biotechnology) and stored at $-80^{\circ} \mathrm{C}$. Protein concentrations were determined using a bicinchoninic acid protein assay kit. Proteins (20 $\mu \mathrm{g} /$ lane) were separated by $10 \%$ SDS-PAGE, transferred to polyvinylidene difluoride membranes and blocked in 5\% fat-free dry milk for $1 \mathrm{~h}$ at room temperature. The membrane was subsequently incubated with UBIAD1 (1:1,000), CoQ10 (1:1,000), Enos (1:1,000) and GAPDH (1:500) primary antibodies at $4^{\circ} \mathrm{C}$ overnight, followed by three washes with Tris-buffered saline with $0.1 \%$ Tween and another incubation with goat-antibody rabbit or goat-anti-mouse horseradish peroxidase conjugated-secondary antibodies $(1: 5,000)$ for $1 \mathrm{~h}$ at room temperature. Protein bands were visualized using an enhanced chemiluminescence detection system (GE Healthcare Life Sciences, Shanghai, China).

Determination of serum and myocardial NO content. Serum samples were collected from animals under anesthesia. Following collection, the serum was stored at $4^{\circ} \mathrm{C}$ for $1 \mathrm{~h}$, and subsequently centrifuged at $625 \mathrm{x} \mathrm{g}$ at $4^{\circ} \mathrm{C}$ for $10 \mathrm{~min}$. Serum and myocardial samples were diluted in PBS to a final concentration of $2 \mu \mathrm{g} / \mu \mathrm{l}$. Samples were boiled for $5 \mathrm{~min}$ and centrifuged at $10,005 \mathrm{x} g$ at $4^{\circ} \mathrm{C}$ for $5 \mathrm{~min}$. Supernatants were collected for NO measurement (Griess method) using the Total Nitric Oxide Measurement kit (cat. no. S0023; Beyotime Insitute of Biotechnology). The standard NO curve was
Table I. Primer sequences used in the quantitative polymerase chain reaction.

\begin{tabular}{|c|c|c|}
\hline Gene name & Direction & Sequence $\left(5^{\prime}-3^{\prime}\right)$ \\
\hline \multirow[t]{2}{*}{ UBIAD1 } & Forward & AACGACTGTCCCGAGCAA \\
\hline & Reverse & CGGCACAACCCACCAA \\
\hline \multirow[t]{2}{*}{$\mathrm{ANF}$} & Forward & AGCCGAGACAGCAAACA \\
\hline & Reverse & GCCTGGGAGCCAAAA \\
\hline \multirow[t]{2}{*}{ CoQ10 } & Forward & GACCATAATGCCTCACC \\
\hline & Reverse & ATGCGTTCATCACCAA \\
\hline \multirow[t]{2}{*}{ eNOS } & Forward & GCAGAGGAGTCCAGCGAACA \\
\hline & Reverse & TGGGTGCTGAGCTGACAGAGT \\
\hline \multirow[t]{2}{*}{ GAPDH } & Forward & GAGGCTCTCTTCCAGCCTTC \\
\hline & Reverse & AGGGTGTAAAAGCAGCTCA \\
\hline
\end{tabular}

UBIAD1, UbiA prenyltransferase domain-containing protein 1; ANF, atrial natriuretic factor; CoQ10, coenzyme Q10; eNOS, endothelial nitric oxide synthase.

prepared by diluting $10 \mathrm{mM} \mathrm{KNO}_{2}$ to $1,10,20$, and $50 \mu \mathrm{mol} / \mathrm{l}$ with PBS ( $\mathrm{pH} 7.4)$, respectively. Supernatants were incubated with lactate dehydrogenase (LDH) Buffer and $\mathrm{LDH}$ at $37^{\circ} \mathrm{C}$ for $30 \mathrm{~min}$. Subsequently, Griess Reagent I and Griess Reagent II were added directly to the above solution and incubated for $10 \mathrm{~min}$ at room temperature, followed by optical measurement. Optical density was measured spectrophotometrically at an absorbance of $540 \mathrm{~nm}$ as previously described (18).

Statistical analysis. Data are expressed as the mean \pm standard deviation. Comparisons between two groups were performed with Student's t-test, and comparisons among multiple groups were performed using one-way analysis of variance followed by Fisher's Least Significant Difference test. All statistical analyses were performed with SPSS 17.0 software (SPSS, Inc., Chicago, IL, USA). $\mathrm{P}<0.05$ was considered to indicate a statistically significant difference.

\section{Results}

SHRs have higher blood pressure, increased cardiac hypertrophic index and impaired cardiac function. Initially, blood pressure and the cardiac hypertrophic index was compared between SHR and WKY rats at 8, 16 and 28 weeks of age. In every age group, SHRs exhibited higher blood pressure (Fig. 1A; ${ }^{*} \mathrm{P}<0.05$ vs. WKY) and increased cardiac hypertrophic index (Fig. 1B; ${ }^{*} \mathrm{P}<0.05$ vs. WKY). Blood pressure in SHRs increased with aging $\left({ }^{\#} \mathrm{P}<0.05\right.$ vs. SHRs at 8 and 16 weeks). In addition, SHRs had a higher LVEDP, as well as a lower LVSP, $+\mathrm{dv} / \mathrm{dt}$ and $-\mathrm{dv} / \mathrm{dt}$, compared with age-matched WKY rats (Table II), indicating cardiac functional impairment in SHRs. Consistent with the above findings, H\&E staining demonstrated disorganized sarcomeres in the hearts of SHRs, compared with well-organized myocardial tissue in age-matched WKY rats (Fig. 1C). Subsequently, myocardial cell apoptosis was assessed with TUNEL staining, which revealed increased apoptosis in SHR 
A

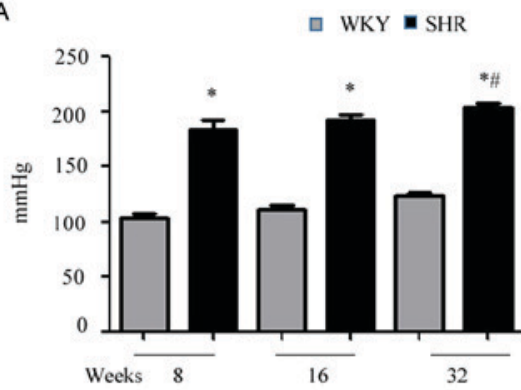

B

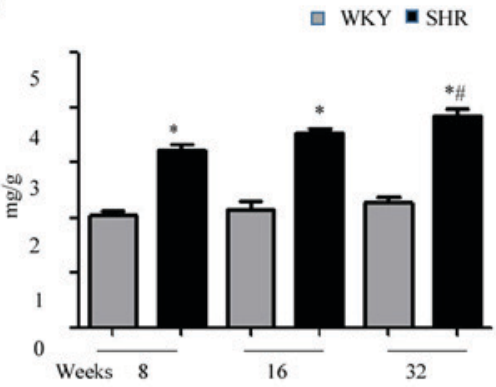

C

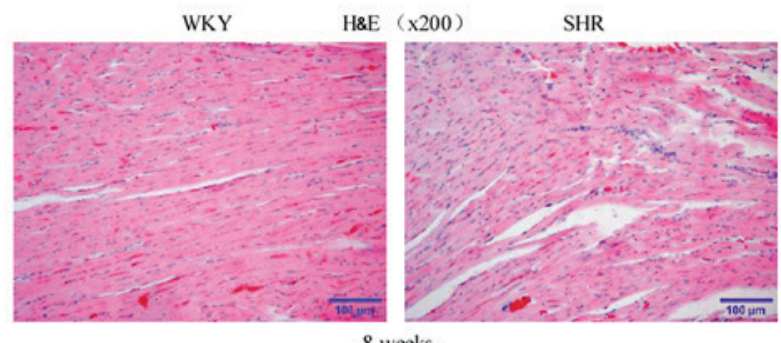

D a

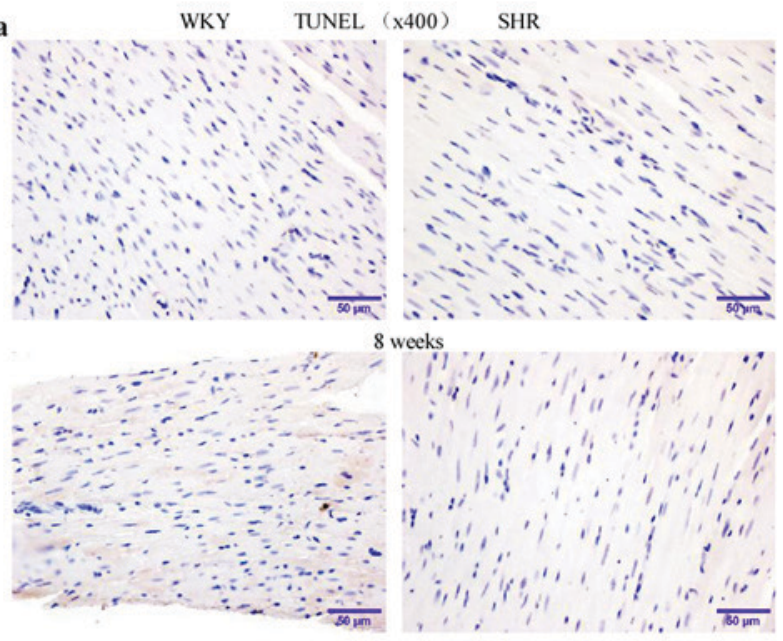

16 weeks
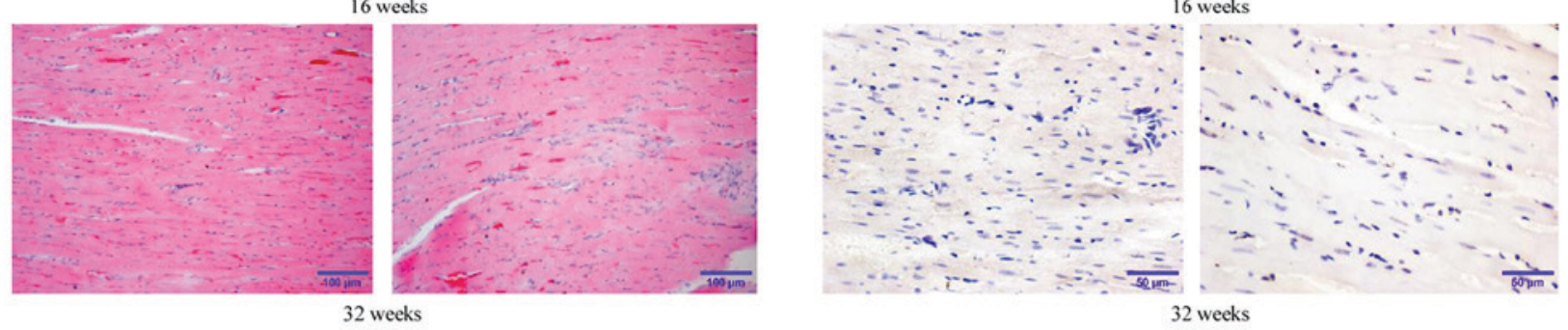

D

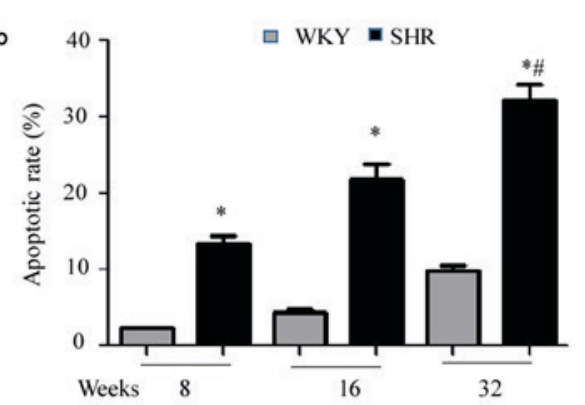

$\mathrm{E}$

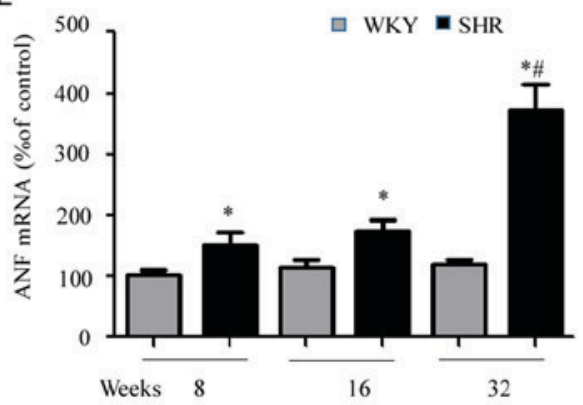

Figure 1. Blood pressure, cardiac hypertrophy and cardiac dysfunction is increased in SHRs. Measurements were obtained at 8, 16 and 32 weeks. (A) Blood pressure of SHR with aging. Blood pressure was measured using a non-invasive tail cut-off multi-channel blood measurement device (B) Cardiac index was determined by the ratio of the left ventricle weight and body weight. (C) H\&E staining was performed on $5 \mu \mathrm{m}$ thick cardiac sections prepared from hearts of SHRs and WKY rats. Magnification, x200. (D-a) TUNEL staining was performed on cardiac sections prepared from SHR and WKY rats. Five randomly selected fields per section were scored and (D-b) the apoptotic rate was calculated. (E) Quantitative polymerase chain reaction was performed to determine ANF expression in the hearts of SHRs and age-matched WKY rats. Each experiment was performed in triplicate. "P<0.05 vs. age matched WKY; ${ }^{\#} \mathrm{P}<0.05$ vs. SHR at 8 weeks. n=8/group. SHRs, spontaneously hypertensive rats; WKY, Wistar-Kyoto; H\&E, hematoxylin \& eosin; TUNEL, terminal deoxynucleotidyl-transferase-mediated dUTP nick end labeling.

hearts, compared with age-matched WKY hearts (Fig. 1D-a and $\left.-\mathrm{b} ;{ }^{*} \mathrm{P}<0.05\right)$. In addition, increased myocardial cell apoptosis was observed with increasing age in SHRs ( ${ }^{\#} \mathrm{P}<0.05$ vs. 8 and 16 weeks). In agreement with the above observations, RT-qPCR demonstrated significant upregulation of atrial natriuretic factor (ANF), a cardiac disease marker, in the hearts of SHRs compared with WKY rats. Furthermore, this upregulation increased with age in SHRs (Fig. 1E; ${ }^{*} \mathrm{P}<0.05$ vs. WKY; ${ }^{\text {P }}<0.05$ vs. 8 and 16 week SHRs). Collectively, these findings supported previous studies $(19,20)$ demonstrating that SHRs exhibit age-associated hypertension and cardiac hypertrophy, as well as impaired cardiac function, accompanied by disorganized myocardium and increased cell death. 
Table II. Cardiac function parameters in SHRs and WKY rats.

A, 8 weeks

\begin{tabular}{lcccc}
\hline Experimental group & LVSP, $\mathrm{mmHg}$ & LVEDP, $\mathrm{mmHg}$ & $+\mathrm{dv} / \mathrm{dt}$ max, $\mathrm{mmHg} / \mathrm{msec}$ & $-\mathrm{dv} / \mathrm{dt} \mathrm{max}, \mathrm{mmHg} / \mathrm{msec}$ \\
\hline WKY & $127 \pm 5.3$ & $2.53 \pm 0.2$ & $5.32 \pm 1.12$ & $5.15 \pm 1.13$ \\
SHR & $97 \pm 3.8^{\mathrm{a}}$ & $6.65 \pm 0.5^{\mathrm{a}}$ & $4.35 \pm 0.72^{\mathrm{a}}$ & $4.12 \pm 0.75^{\mathrm{a}}$ \\
\hline
\end{tabular}

B, 16 weeks

\begin{tabular}{lcccc}
\hline Experimental group & LVSP, $\mathrm{mmHg}$ & LVEDP, $\mathrm{mmHg}$ & $+\mathrm{dv} / \mathrm{dt}$ max, $\mathrm{mmHg} / \mathrm{msec}$ & $-\mathrm{dv} / \mathrm{dt} \mathrm{max}, \mathrm{mmHg} / \mathrm{msec}$ \\
\hline WKY & $122 \pm 4.6$ & $2.97 \pm 0.5$ & $4.95 \pm 0.95$ & $4.44 \pm 0.94$ \\
SHR & $91 \pm 3.6^{\mathrm{a}}$ & $7.43 \pm 0.6^{\mathrm{a}}$ & $3.79 \pm 0.68^{\mathrm{a}}$ & $3.38 \pm 0.71^{\mathrm{a}}$ \\
\hline
\end{tabular}

C, 28 weeks

\begin{tabular}{lcccc}
\hline Experimental group & LVSP, mmHg & LVEDP, $\mathrm{mmHg}$ & $+\mathrm{dv} / \mathrm{dt} \max , \mathrm{mmHg} / \mathrm{msec}$ & $-\mathrm{dv} / \mathrm{dt} \mathrm{max}, \mathrm{mmHg} / \mathrm{msec}$ \\
\hline WKY & $118 \pm 3.7$ & $3.74 \pm 0.8$ & $4.56 \pm 0.86$ & $4.37 \pm 0.79$ \\
SHR & $86 \pm 2.9^{\mathrm{a}, \mathrm{b}}$ & $8.37 \pm 0.5^{\mathrm{a}, \mathrm{b}}$ & $2.56 \pm 0.62^{\mathrm{a}, \mathrm{b}}$ & $2.32 \pm 0.58^{\mathrm{a}, \mathrm{b}}$ \\
\hline
\end{tabular}

${ }^{\mathrm{a}} \mathrm{P}<0.05$ vs. WKY group; ${ }^{\mathrm{b}} \mathrm{P}<0.05$ vs. 8 and 16 week SHR groups. LVSP, left ventricular systolic pressure; LVEDP, left ventricular end diastolic pressure; WKY, Wistar-Kyoto rats; SHR, spontaneously hypertensive rats.

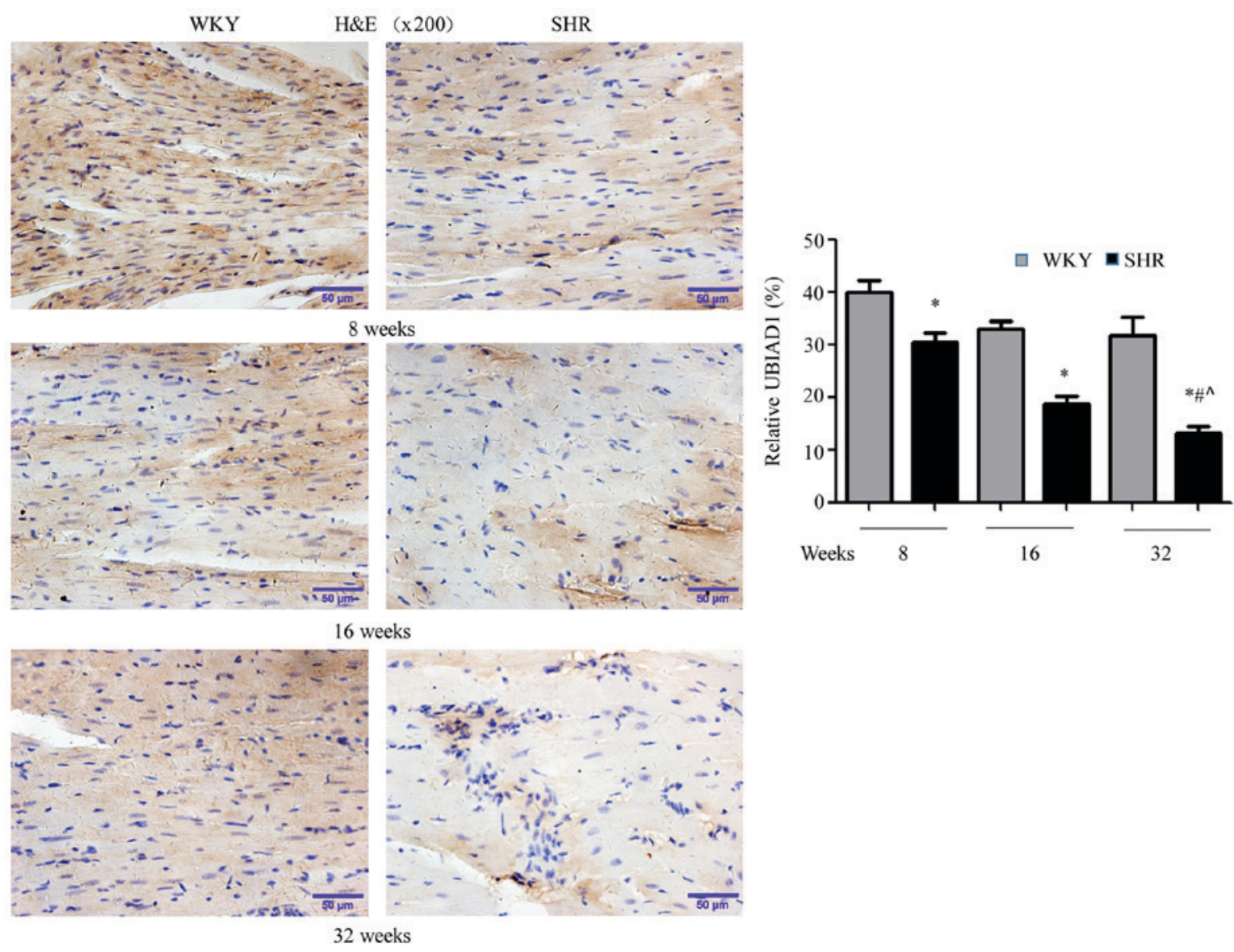

Figure 2. UBIAD1 expression is reduced in SHR hearts. Immunohistochemical analysis was performed using a specific antibody against UBIAD1 on cardiac sections prepared from SHRs and age-matched WKY rats, and the results were statistically analyzed. In total, five randomly selected fields were scored and quantified with Image-Pro Plus 5.0. " $\mathrm{P}<0.05$ vs. age matched WKY rats; ${ }^{*} \mathrm{P}<0.05$ vs. SHRs at 8 weeks; ${ }^{\wedge} \mathrm{P}<0.05$ vs. SHR at 16 weeks. $\mathrm{n}=8 /$ group. SHRs, spontaneously hypertensive rats; WKY, Wistar-Kyoto; UBIAD1, UbiA prenyltransferase containing 1. 
A

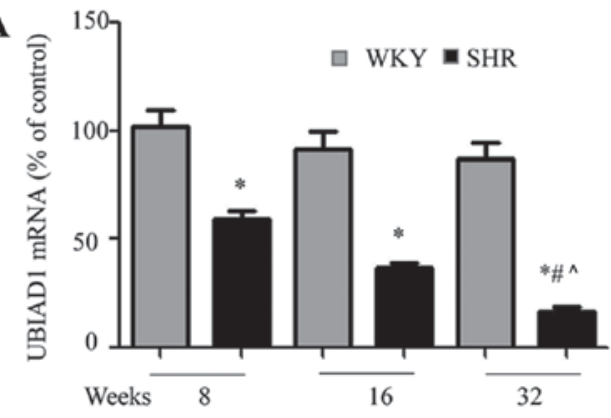

C

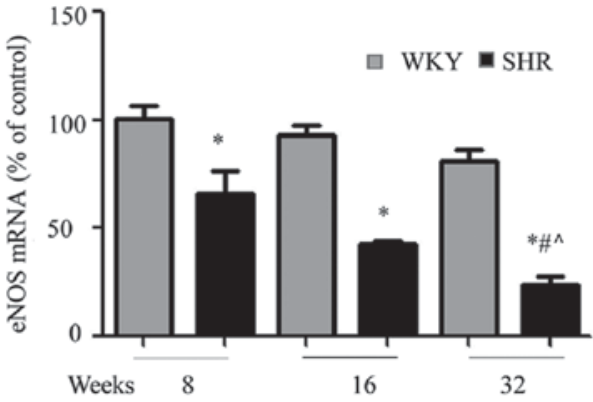

B

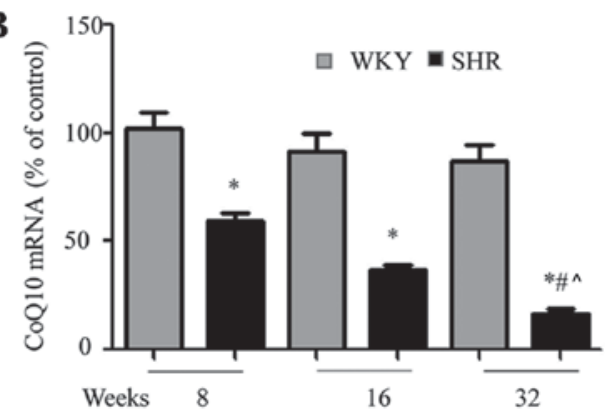

D

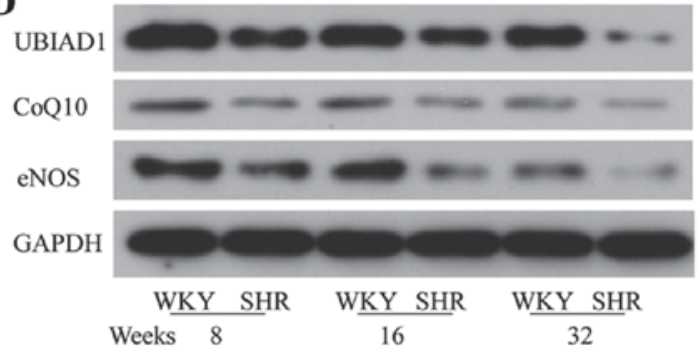

Figure 3. UBIAD1, CoQ10 and eNOS expression is decreased in SHR hearts. (A) Quantitative polymerase chain reaction demonstrated that UBIAD1, (B) CoQ10 and (C) eNOS mRNA expression was decreased in SHR hearts, compared with age-matched WKY rat hearts. (D) Western blotting demonstrated that UBIAD1, CoQ10 and eNOS protein expression was decreased in SHR hearts, compared with WKY rat hearts. Each experiment was performed in triplicate. ${ }^{*} \mathrm{P}<0.05$ vs. age matched WKY rats; ${ }^{\#} \mathrm{P}<0.05$ vs. SHRs at 8 weeks; ${ }^{\wedge} \mathrm{P}<0.05$ vs. SHR at 16 weeks. UBIAD1, UbiA prenyltransferase containing $1 ;$ CoQ10, coenzyme Q10; eNOS, endothelial nitric oxide synthase; SHRs, spontaneously hypertensive rats; WKY, Wistar-Kyoto.
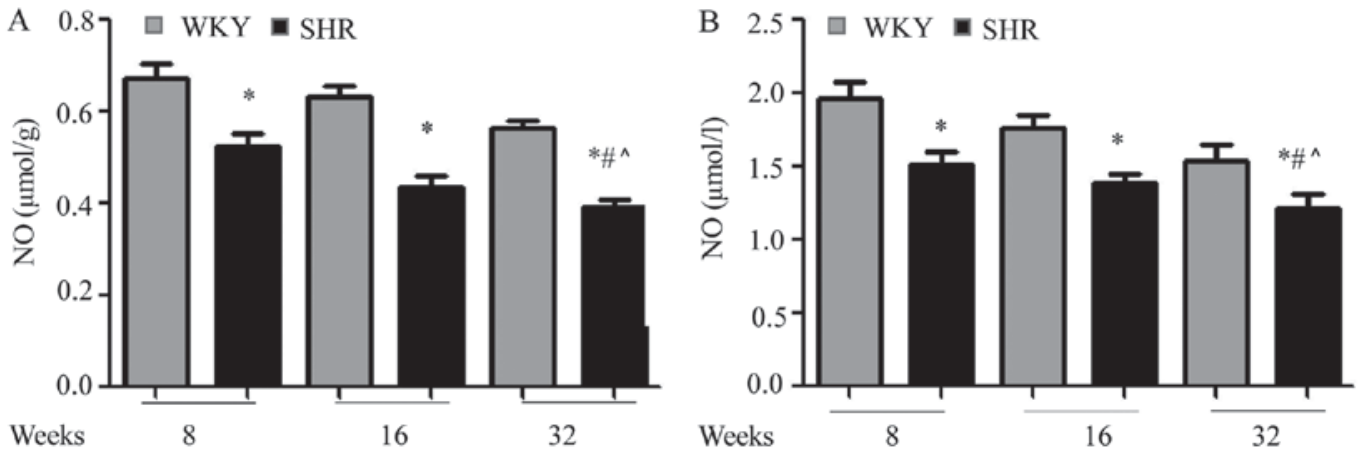

Figure 4. Decreased circulating and myocardial NO in SHRs. Reduced NO was detected in the (A) myocardial tissue and (B) serum of SHRs, compared with age-matched WKY rats. ${ }^{*} \mathrm{P}<0.05$ vs. age matched WKY rats; ${ }^{\#} \mathrm{P}<0.05$ vs. SHRs at 8 weeks; ${ }^{\wedge} \mathrm{P}<0.05$ vs. SHR at 16 weeks. $\mathrm{n}=3 /$ group.

UBIAD1 expression decreases in SHR hearts. To determine the potential involvement of UBIAD1 in hypertensive cardiac hypertrophy, the expression of UBIAD1 in the hearts of SHR and age-matched WKY rats was initially evaluated by immunohistochemistry. As presented in Fig. 2, UBIAD1 staining was weaker in SHR hearts compared with WKY hearts, at each age examined ( $\mathrm{P}<0.05$ vs. WKY). Additionally, UBIAD1 expression in SHR hearts decreased with aging ( ${ }^{\#} \mathrm{P}<0.05$ vs. SHR at 8 and 16 weeks). These data demonstrated that UBIAD1 expression is downregulated in SHR hearts, compared with age-matched WKY hearts, and that this downregulation increased over time.

UBIAD1, CoQ10 and eNOS expression decrease in SHR hearts. UBIAD1 has been observed to be involved in mediating CoQ10 activity, which mediates eNOS expression (5). Therefore, the gene and protein expression of CoQ10, eNOS and UBIAD1 was measured using RT-qPCR and western blotting, respectively. In line with the immunohistochemical findings, UBIAD1 mRNA expression was downregulated in SHR hearts compared with WKY rat hearts, and this downregulation was age-dependent in SHRs (Fig. $3 \mathrm{~A}$; ${ }^{*} \mathrm{P}<0.05$ vs. WKY; ${ }^{\text {P }}<0.05$ vs. SHRs at 8 and 16 weeks). CoQ10 (Fig. 3B) and eNOS (Fig. 3C) expression was significantly lower in the SHR hearts compared to WKY hearts at 8, 16 and 28 weeks $\left(\mathrm{P}<0.05\right.$ vs. WKY; ${ }^{\#} \mathrm{P}<0.05$ vs. SHRs at 8 and 16 weeks $)$. Furthermore, a similar trend in UBIAD1, CoQ10 and eNOS protein expression was observed (Fig. 3D). Taken together, these results demonstrated that downregulated UBIAD1 expression in SHR hearts was associated with decreased CoQ10 and eNOS expression.

Circulating and myocardial NO are decreased in SHR. As eNOS is an important regulator of NO production (21), the NO content in the serum and myocardial tissue of SHR and WKY rats was determined. As present in Fig. 4A, it was 
demonstrated that NO content was decreased in SHR hearts, compared with age-matched WKY hearts ( $\mathrm{P}<0.05$ vs. WKY), in agreement with the eNOS results. The NO content in the SHR hearts also decreased with aging $\left({ }^{\#} \mathrm{P}<0.05\right.$ vs. SHRs at 8 and 16 weeks). Further, it was demonstrated that serum NO levels were significantly lower in SHRs, compared with WKY rats (Fig. 4B; ${ }^{*} \mathrm{P}<0.05$ vs. WKY), and that NO was further reduced with aging ( ${ }^{\#} \mathrm{P}<0.05$ vs. SHRs at 8 and 16 weeks). Therefore, it was demonstrated that NO expression in the hearts and serum of SHRs was substantially downregulated, and that this effect further increases with aging.

\section{Discussion}

Although UBIAD1 has been reported to be involved in a number of human diseases $(1,2,4-6)$, its potential contribution to cardiovascular disorders remains unclear. In the present study, it was confirmed that SHR exhibited age-associated increases in blood pressure and myocardial apoptosis, accompanied by cardiac dysfunction deterioration. Furthermore, increased expression of ANF, a cardiac disease marker, was detected. Immunohistochemical analysis, RT-qPCR and western blotting also revealed decreased UBIAD1 expression in SHR hearts, compared with the control group. This decrease was associated with decreased myocardial CoQ10 and eNOS expression. In addition, serum and myocardial tissue NO expression levels were significantly lower in SHRs. Furthermore, expression of the aforementioned factors decreased in SHRs in an age-dependent manner. Given the role of UBIAD1 in eNOS and CoQ10 signaling regulation (5), the data obtained in the present study indicated that UBIAD1 had critical functions in decreasing eNOS, CoQ10 and NO expression in hypertensive cardiac hypertrophy.

The principal finding of the present study was that myocardial UBIAD1 expression was significantly downregulated in SHRs, and that this downregulation was associated with age. A previous study suggested that high UBIAD1 expression in human heart tissue was indicative of its potential role in heart disease (7). Indeed, as a biosynthetic enzyme for both vitamin K2 and MK-4 (6), a UBIAD1 mutant caused a number of phenotypes, including cardiac edema in zebrafish, which were rescued by re-repressing wild-type human UBIAD1 (7). $\mathrm{UBIAD}^{-/-}$mouse embryos die before embryonic day 10.5 ; however, it remains unclear if these mutants exhibited cardiac structural defects prior to death (22). To the best of our knowledge, the present study was the first to demonstrate that UBIAD1 expression is decreased in hypertension-associated hypertrophic hearts. This merits further examination, in order to determine whether UIBAD1 expression is altered in other myocardial disease states, including ischemia/reperfusion and pressure-overload induced cardiac hypertrophy. In addition, it would be of great interest to investigate if UBIAD1 expression is altered in human cardiac muscle disorders and heart failure.

Another question that requires addressing is how UBIAB1 expression is regulated in hearts. A recent study suggested that transcriptional repressor protein YY1, which is additionally a ubiquitously expressed factor like UBIAB1, is a positive regulator of UBIAD1 expression (23). YY1 serves an important role in early cardiac development, cardiac muscle disorders and heart failure (24-26). YY1 additionally offers protection against pathological hypertrophy (27). Therefore, the role of YY1 in regulating UBIAD1 expression in hypertrophic myocardium warrants further research.

In the present study, while a decrease in UBIAD1 expression in the SHRs was observed, it remains unclear whether this decrease was the consequence of hypertrophy or if it was an underlying cause. It could also not be concluded that decreased UBIAD1 expression directly exacerbated cardiac dysfunction in SHRs with age. In the future, the effects of UBIAD1 on the earlier stages of SHR prior to the development of overt cardiac hypertrophy will be used, as well as a cardiac-specific UBIAD1 knockout to further address these questions.

In addition to its role in vitamin $\mathrm{K}$ synthesis, UBIAD1 catalyzes the biosynthesis of CoQ10 and mediates eNOS activity in zebrafish and humans. The role for CoQ10 in cardiac hypertrophy and heart failure has been well investigated clinically and in animal models (28). CoQ10 expression is deficient in a number of cardiac diseases (29). Consistently, CoQ10 pretreatment is thought to be protective against isoproterenol-induced rat model of cardiac hypertrophy (30), and previous clinical studies have demonstrated the beneficial effects of CoQ10 administration in patients with heart failure $(31,32)$. In the present study, it was demonstrated that CoQ10 expression was substantially decreased in hypertension-induced hypertrophic myocardial tissue, which was accompanied by decreased expression of its downstream effector, eNOS $(33,34)$. It has been well documented that NO is catalyzed by eNOS, and is released by vascular endothelial cells and cardiomyocytes where it functions as a vasodilator (11). eNOS is widely expressed in vascular endothelial cells, cardiomyocytes and platelets and serves an important role in the maintenance of cardiovascular homeostasis, predominantly through regulating NO expression (35). NO has a variety of biological activities, including platelet aggregation inhibition, cardiac function regulation, vascular smooth muscle relaxation (36). $\mathrm{NO}$ has critical functions in vascular tone maintenance and blood pressure regulation, as evidenced by the findings that inhibition of NO synthesis increases blood pressure in healthy humans (37). It has been reported that hypertensive patients have decreased serum NO levels and eNOS gene expression (38). In line with previous findings, the present study demonstrated that circulating and myocardial NO levels were decreased in SHRs compared with age-matched control WKY rats, and that this decrease was exacerbated with aging. Given the role of UBIAD1 in mediating CoQ10 activity, the findings of the present study collectively pointed to the potential contribution of the UBIAD1-CoQ10-eNOS-NO axis in the pathogenesis of hypertensive cardiac hypertrophy, by which reduced levels of UBIAD1 resulted in insufficient expression of CoQ10, subsequently diminishing eNOS expression and NO levels. This hypothesis should be further investigated in future studies.

Previous studies have suggested that UBIAD1 regulates apoptosis through multiple mechanisms. For instance, UBIAD1 was reported to promote apoptosis through mediating Golgi function in a number of human cancer cell lines (39), and another study indicated that UBIAD1 induces apoptosis in bladder tumor cells through regulating cellular cholesterol (40). Consistent with the above findings, the present study revealed that decreased UBIAD1 expression was accompanied 
by increased apoptosis in SHR hearts. However, a direct link between these two factors remains to be established.

Certain limitations of the present study require acknowledgement. As mentioned above, future studies must be performed to reveal the causative relationship between decreased UBIAD1 expression and the pathogenesis of hypertension-related cardiac hypertrophy. One way to address this would be to rescue cardiac function and hypertrophy by administering UBIAD1 to SHRs. Another limitation was that a direct link between decreased UBIAD1 expression and decreased CoQ10 and eNOS levels was not established. Given that a previous study using UBIAD1 knockout mice suggested that UBIAD1 may not be a major regulator of CoQ10 expression in mice (22), establishment of such direct evidence is important to understand the exact function of UBIAD1 pin the development and progression of hypertensive cardiac hypertrophy.

In conclusion, it was demonstrated that UBIAD1 expression was decreased in SHR hearts in an age-dependent manner, compared with those of age-matched controls. Altered UBIAD1 expression was accompanied by reduced CoQ10, eNOS, and NO expression in SHRs. Given the well-recognized benefits offered by CoQ10 for cardiovascular diseases, UBIAD1 may represent a potential therapeutic target for the clinical treatment of hypertension-induced cardiac hypertrophy.

\section{Acknowledgements}

The authors would like to thank Mrs Song Ying of the Central Laboratory of the First Affiliated Hospital of Jinzhou Medical University (Jinzhou, China) for the experimental guidance and technical support.

\section{Funding}

The present study was supported by the Youth Project of Liaoning Provincial Department of Education (grant no. JYTQN201714).

\section{Availability of data and materials}

The datasets used and/or analyzed during the present study are available from the corresponding author on reasonable request.

\section{Authors' contributions}

BY performed the experiments. JW designed the experiments and supervised the study. All authors read and approved the final manuscript.

\section{Ethics approval and consent to participate}

All animal protocols complied with and were approved by the Ethics Committee of Jinzhou Medical University (Jinzhou, China).

\section{Patient consent for publication}

Not applicable.

\section{Competing interests}

The authors declare that they have no competing interests.

\section{References}

1. McGarvey TW, Nguyen T, Puthiyaveettil R, Tomaszewski JE and Malkowicz SB: TERE1, a novel gene affecting growth regulation in prostate carcinoma. Prostate 54: 144-155, 2003.

2. Orr A, Dubé MP, Marcadier J, Jiang H, Federico A, George S, Seamone C, Andrews D, Dubord P, Holland S, et al: Mutations in the UBIAD1 gene, encoding a potential prenyltransferase, are causal for Schnyder crystalline corneal dystrophy. PLoS One 2: e685, 2007.

3. McGarvey TW, Nguyen T, Tomaszewski JE, Monson FC and Malkowicz SB: Isolation and characterization of the TERE1 gene, a gene down-regulated in transitional cell carcinoma of the bladder. Oncogene 20: 1042-1051, 2001.

4. Nickerson ML, Kostiha BN, Brandt W, Fredericks W, Xu KP, Yu FS, Gold B, Chodosh J, Goldberg M, Lu DW, et al: UBIAD1 mutation alters a mitochondrial prenyltransferase to cause Schnyder corneal dystrophy. PLoS One 5: e10760, 2010.

5. Mugoni V, Postel R, Catanzaro V, De Luca E, Turco E, Digilio G, Silengo L, Murphy MP, Medana C, Stainier DY, et al: Ubiad1 is an antioxidant enzyme that regulates eNOS activity by CoQ10 synthesis. Cell 152: 504-518, 2013.

6. Nakagawa K, Hirota Y, Sawada N, Yuge N, Watanabe M, Uchino Y, Okuda N, Shimomura Y, Suhara Y and Okano T: Identification of UBIAD1 as a novel human menaquinone-4 biosynthetic enzyme. Nature 468: 117-121, 2010.

7. Hegarty JM, Yang H and Chi NC: UBIAD1-mediated vitamin $\mathrm{K} 2$ synthesis is required for vascular endothelial cell survival and development. Development 140: 1713-1719, 2013.

8. Tousoulis D, Kampoli AM, Tentolouris C, Papageorgiou N and Stefanadis C: The role of nitric oxide on endothelial function. Curr Vasc Pharmacol 10: 4-18, 2012.

9. Vos M, Esposito G, Edirisinghe JN, Vilain S, Haddad DM, Slabbaert JR, Van Meensel S, Schaap O, De Strooper B, Meganathan $\mathrm{R}$, et al: Vitamin $\mathrm{K}_{2}$ is a mitochondrial electron carrier that rescues pink1 deficiency. Science 336: 1306-1310, 2012.

10. Xia Y, Wei X, Wu S, Wang B, Wang X and Hong L: Down-regulation of TERE1/UBIAD1 activated Ras-MAPK signalling and induced cell proliferation. Cell Biol Int Rep 17: e00005, 2010.

11. Zhan CD: The role of nitric oxide in the prevention of myocardial hypertrophic response and its mechanisms. Sheng Li Ke Xue Jin Zhan 31: 322-324, 2000 (In Chinese).

12. Wollert KC and Drexler H: Regulation of cardiac remodeling by nitric oxide: Focus on cardiac myocyte hypertrophy and apoptosis. Heart Fail Rev 7: 317-325, 2002.

13. Ozaki M, Kawashima S, Yamashita T, Hirase T, Ohashi Y, Inoue N, Hirata K and Yokoyama M: Overexpression of endothelial nitric oxide synthase attenuates cardiac hypertrophy induced by chronic isoproterenol infusion. Circ J 66: 851-856, 2002.

14. Flaherty MP, Brown M, Grupp IL, Schultz JE, Murphree SS and Jones WK: eNOS deficient mice develop progressive cardiac hypertrophy with altered cytokine and calcium handling protein expression. Cardiovasc Toxicol 7: 165-177, 2007.

15. Brede M, Roell W, Ritter O, Wiesmann F, Jahns R, Haase A, Fleischmann BK and Hein L: Cardiac hypertrophy is associated with decreased eNOS expression in angiotensin AT2 receptor-deficient mice. Hypertension 42: 1177-1182, 2003.

16. Yan B, Sun Y and Wang J: Depletion of ubiA prenyltransferase domain containing 1 expression promotes angiotensin II-induced hypertrophic response in AC16 human myocardial cells via modulating the expression levels of coenzyme Q10 and endothelial nitric oxide synthase. Mol Med Rep 16: 6910-6915, 2017.

17. Livak KJ and Schmittgen TD: Analysis of relative gene expression data using real-time quantitative PCR and the $2^{-\Delta \Delta C_{\mathrm{T}}}$ method. Methods 25: 402-408, 2001.

18. Ridnour LA, Sim JE, Hayward MA, Wink DA, Martin SM, Buettner GR and Spitz DR: A spectrophotometric method for the direct detection and quantitation of nitric oxide, nitrite, and nitrate in cell culture media. Anal Biochem 281: 223-229, 2000. 
19. Nordborg C and Johansson BB: Morphometric study on cerebral vessels in spontaneously hypertensive rats. Stroke 11: 266-270, 1980.

20. Diez J, Panizo A, Hernández M, Vega F, Sola I, Fortuño MA and Pardo J: Cardiomyocyte apoptosis and cardiac angiotensin-converting enzyme in spontaneously hypertensive rats. Hypertension 30: 1029-1034, 1997.

21. Förstermann U and Sessa WC: Nitric oxide synthases: Regulation and function. Eur Heart J 33: 829-837, 837a-837d, 2012

22. Nakagawa K, Sawada N, Hirota Y, Uchino Y, Suhara Y, Hasegawa $\mathrm{T}$, Amizuka N, Okamoto $\mathrm{T}$, Tsugawa $\mathrm{N}$, Kamao $\mathrm{M}$, et al: Vitamin $\mathrm{K}_{2}$ biosynthetic enzyme, UBIAD1 is essential for embryonic development of mice. PLoS One 9: e104078, 2014

23. Funahashi N, Hirota Y, Nakagawa K, Sawada N, Watanabe M, Suhara Y and Okano T: YY1 positively regulates human UBIAD1 expression. Biochem Biophys Res Commun 460: 238-244, 2015.

24. Beketaev I, Zhang Y, Kim EY, Yu W, Qian L and Wang J: Critical role of YY1 in cardiac morphogenesis. Dev Dyn 244: 669-680, 2015.

25. Stauffer BL, Dockstader K, Russell G, Hijmans J, Walker L, Cecil M, Demos-Davies K, Medway A, McKinsey TA and Sucharov CC: Transgenic over-expression of YY1 induces pathologic cardiac hypertrophy in a sex-specific manner. Biochem Biophys Res Commun 462: 131-137, 2015.

26. Sucharov CC, Dockstader K and McKinsey TA: YY1 protects cardiac myocytes from pathologic hypertrophy by interacting with HDAC5. Mol Biol Cell 19: 4141-4153, 2008.

27. Sucharov CC, Mariner P, Long C, Bristow M and Leinwand L: Yin Yang 1 is increased in human heart failure and represses the activity of the human alpha-myosin heavy chain promoter. J Biol Chem 278: 31233-31239, 2003.

28. Kumar A, Kaur H, Devi P and Mohan V: Role of coenzyme Q10 (CoQ10) in cardiac disease, hypertension and Meniere-like syndrome. Pharmacol Ther 124: 259-268, 2009.

29. Muraro PA, Vergelli M, Kalbus M, Banks DE, Nagle JW Tranquill LR, Nepom GT, Biddison WE, McFarland HF and Martin R: Immunodominance of a low-affinity major histocompatibility complex-binding myelin basic protein epitope (residues 111-129) in HLA-DR4 $(\mathrm{B} 1 * 0401)$ subjects is associated with a restricted T cell receptor repertoire. J Clin Invest 100: 339-349, 1997.
30. Ghule AE, Kulkarni CP, Bodhankar SL and Pandit VA: Effect of pretreatment with coenzyme Q10 on isoproterenol-induced cardiotoxicity and cardiac hypertrophy in rats. Curr Ther Res Clin Exp 70: 460-471, 2009.

31. Fotino AD, Thompson-Paul AM and Bazzano LA: Effect of coenzyme $\mathrm{Q}_{10}$ supplementation on heart failure: A meta-analysis. Am J Clin Nutr 97: 268-275, 2013.

32. Sharma A, Fonarow GC, Butler J, Ezekowitz JA and Felker GM: Coenzyme Q10 and heart failure: A state-of-the-art review. Circ Heart Fail 9: e002639, 2016.

33. Chew GT and Watts GF: Coenzyme Q10 and diabetic endotheliopathy: Oxidative stress and the 'recoupling hypothesis'. QJM 97: 537-548, 2004

34. Tsai KL, Huang YH, Kao CL, Yang DM, Lee HC, Chou HY, Chen YC, Chiou GY, Chen LH, Yang YP, et al: A novel mechanism of coenzyme Q10 protects against human endothelial cells from oxidative stress-induced injury by modulating NO-related pathways. J Nutr Biochem 23: 458-468, 2012.

35. Forstermann U and Münzel T: Endothelial nitric oxide synthase in vascular disease: From marvel to menace. Circulation 113: 1708-1714, 2006.

36. Bian K, Doursout MF and Murad F: Vascular system: Role of nitric oxide in cardiovascular diseases. J Clin Hypertens 10 304-310, 2008.

37. Kang JH, Wiggs JL, Rosner BA, Haines J, Abdrabou W and Pasquale LR: Endothelial nitric oxide synthase gene variants and primary open-angle glaucoma: Interactions with hypertension, alcohol intake and cigarette smoking. Arch Ophthalmol 129: 773-780, 2011.

38. Li Q, Youn JY and Cai H: Mechanisms and consequences of endothelial nitric oxide synthase dysfunction in hypertension. J Hypertens 33: 1128-1136, 2015.

39. Wang X, Wang D, Jing P, Wu Y, Xia Y, Chen M and Hong L: A novel Golgi retention signal RPWS for tumor suppressor UBIAD1. PLoS One 8: e72015, 2013.

40. Fredericks WJ, McGarvey T, Wang H, Lal P, Puthiyaveettil R, Tomaszewski J, Sepulveda J, Labelle E, Weiss JS, Nickerson ML, et al: The bladder tumor suppressor protein TERE1 (UBIAD1) modulates cell cholesterol: Implications for tumor progression. DNA Cell Biol 30: 851-864, 2011. 